\title{
BMC Women's Health reviewer acknowledgement 2015
}

Nawsheen Boodhun

\section{Contributing reviewers}

The editors of BMC Women's Health would like to thank all our reviewers who have contributed to the journal in Volume 15 (2015).

Julie Abayomi

UK

Misra Abdulahi

Ethiopia

Gedefaw Abeje

Ethiopia

Philip Adongo

Ghana

Kelvin Afrashtehfar

Canada

Maryam Ahmadian

Malaysia

Leonard Ajah

Nigeria

Tomi Akinyemiju

USA

Angela Akol

Uganda

Melissa Aldrich

USA

Habiba Isse Ali

United Arab Emirates

Nisreen Alwan

UK

Eyitope Amu

Nigeria
Sari Andajani

New Zealand

Troy Andersen

USA

Eleanor Anderson

USA

Ewa Andersson

Sweden

Muhammad Arif

USA

Ludwin Artur

Poland

Ian Askew

Kenya

Nega Assefa

Ethiopia

Lou Atkinson

UK

Jodie Avery

Australia

Susan Ayers

UK

Shawky Badawy

USA

Donna Baird

USA

\author{
Ana Paula Barbosa \\ Portugal \\ Lesley Barclay \\ Australia \\ Janine Barden-O'Fallon \\ USA
}

\section{Farihan Barghouti}

Jordan

Arunodaya Barman
Malaysia

Rebecca Barr

UK
Elizabeth Barrett-Connor
USA

Hyam Bashour

Syria

Partha Basu

India

Majed Bata

Jordan

Moses Bateganya

Uganda

Ronald Battt

USA

Wafa Bawab

Lebanon

Correspondence: Nawsheen.Boodhun@biomedcentral.com

BioMed Central, Floor 6, 236 Gray's Inn Road, London WC1X 8HB, UK 
Angela Bazzi
USA

Aris Bechlioulis

Greece

Bei Bei

Australia

Ruud Bekkers

Netherlands

Tefera Belachew

Ethiopia

Laura Benaglia

Italy

Elizabeth Bertone-Johnson

USA

Jane Bertrand

USA

Rebecca Beyda

USA

Premananda Bharati

India

Neerja Bhatla

India

Nazan Bilgel

Turkey

Lorena Binfa

Chile

Kirsten Black

Australia

Tina Bloom

USA

Eric Blyth

UK

Daniel Bogale

Ethiopia

Jesper Bonde

Denmark

Matteo Bonzini

Italy

Remko Bosgraaf

Netherlands

Massimo Bracci

Italy

Daniel Breitkopf

USA
Molly Brewer

USA

Hilary Brown

Canada

Katherine Brown

UK

Joelle Brown

USA

Lauro Bucchi

Italy

Carol Bugge

UK

Thanh Bui

Viet Nam

Ann Burchell

Canada

Henry Burger

Australia

Helene Caillon

France

Elyce Cardonick

USA

Mark Carey

Canada

Marcia Carneiro

Brazil

Pilar Carrasco-Garrido

Spain

Rosario Castaño

Spain

Alejandra Castanon

UK

Patrick Catoire

France

Mario Cavagna

USA

Christina Chambers

USA

Arundhati Char

India

Dongbao Chen

USA

Donna Chizen

Canada
Donna Chung

Australia

Crystal Clark

USA

Kelly Cobey

Canada

Richard Cone

USA

Xavier Corbella Virós

Spain

Ali Kagan Coskun

Turkey

Jeremy Couper

Australia

Annie Cushing

UK

Michele Da Broi

Brazil

Rada Dagher

USA

Stefania D'Angelo

UK

Chantal Daumerie

Belgium

Melissa A. Davey-Rothwell

USA

Matthias David

Germany

Karen Davies

UK

Ayesha De Costa

Sweden

Mary De Silva

UK

Amanda Deeks

Australia

Thérèse Delvaux

Benin

Lynette Denny

South Africa

Haryana Dhillon

Australia

Cyril Dim

Nigeria 


\begin{tabular}{|c|c|c|}
\hline $\begin{array}{l}\text { Mai Do } \\
\text { USA }\end{array}$ & $\begin{array}{l}\text { Mary Fisher } \\
\text { USA }\end{array}$ & $\begin{array}{l}\text { Lisa Haddad } \\
\text { USA }\end{array}$ \\
\hline $\begin{array}{l}\text { Yusuf Cetin Doganer } \\
\text { Turkey }\end{array}$ & $\begin{array}{l}\text { Arthur Fleischer } \\
\text { USA }\end{array}$ & $\begin{array}{l}\text { Maria Hadjifrangiskou } \\
\text { USA }\end{array}$ \\
\hline $\begin{array}{l}\text { Wybo Dondorp } \\
\text { Netherlands }\end{array}$ & $\begin{array}{l}\text { Andrew Flower } \\
\text { UK }\end{array}$ & $\begin{array}{l}\text { Helen Haines } \\
\text { Australia }\end{array}$ \\
\hline $\begin{array}{l}\text { Tam Truong Donnelly } \\
\text { Canada }\end{array}$ & $\begin{array}{l}\text { Randi Foraker } \\
\text { USA }\end{array}$ & $\begin{array}{l}\text { Vivienne Hanley } \\
\text { UK }\end{array}$ \\
\hline $\begin{array}{l}\text { Frances Doran } \\
\text { Australia }\end{array}$ & $\begin{array}{l}\text { Caren Frost } \\
\text { USA }\end{array}$ & $\begin{array}{l}\text { Estrid Stæhr Hansen } \\
\text { Denmark }\end{array}$ \\
\hline $\begin{array}{l}\text { Alison Drake } \\
\text { USA }\end{array}$ & $\begin{array}{l}\text { Franco Fulciniti } \\
\text { Italy }\end{array}$ & $\begin{array}{l}\text { Lisa Hanson } \\
\text { USA }\end{array}$ \\
\hline $\begin{array}{l}\text { Kristin Dunkle } \\
\text { USA }\end{array}$ & $\begin{array}{l}\text { Qinglei Gao } \\
\text { China }\end{array}$ & $\begin{array}{l}\text { Diane Harper } \\
\text { USA }\end{array}$ \\
\hline $\begin{array}{l}\text { Christine East } \\
\text { Australia }\end{array}$ & $\begin{array}{l}\text { Hongshan Ge } \\
\text { China }\end{array}$ & $\begin{array}{l}\text { Melissa Harris } \\
\text { Australia }\end{array}$ \\
\hline $\begin{array}{l}\text { Gillian Eastgate } \\
\text { Australia }\end{array}$ & $\begin{array}{l}\text { Caitlin Gerdts } \\
\text { USA }\end{array}$ & $\begin{array}{l}\text { Laura Harris } \\
\text { USA }\end{array}$ \\
\hline $\begin{array}{l}\text { Gillian Einstein } \\
\text { Canada }\end{array}$ & $\begin{array}{l}\text { Paolo Giorgi Rossi } \\
\text { Italy }\end{array}$ & $\begin{array}{l}\text { Emily Harville } \\
\text { USA }\end{array}$ \\
\hline $\begin{array}{l}\text { Sebastian Eliason } \\
\text { Ghana }\end{array}$ & $\begin{array}{l}\text { Nancy Glass } \\
\text { USA }\end{array}$ & $\begin{array}{l}\text { Abigail Hatcher } \\
\text { South Africa }\end{array}$ \\
\hline $\begin{array}{l}\text { Khalifa Elmusharaf } \\
\text { Sudan }\end{array}$ & $\begin{array}{l}\text { Jeannine Goetz } \\
\text { USA }\end{array}$ & $\begin{array}{l}\text { Kathy Hegadoren } \\
\text { Canada }\end{array}$ \\
\hline $\begin{array}{l}\text { Karen Ertel } \\
\text { USA }\end{array}$ & $\begin{array}{l}\text { John Gohagan } \\
\text { USA }\end{array}$ & $\begin{array}{l}\text { Ingrid Hellstrom } \\
\text { Sweden }\end{array}$ \\
\hline $\begin{array}{l}\text { Cam Escoffery } \\
\text { USA }\end{array}$ & $\begin{array}{l}\text { Mara Goldman } \\
\text { USA }\end{array}$ & $\begin{array}{l}\text { Frans Helmerhorst } \\
\text { Netherlands }\end{array}$ \\
\hline $\begin{array}{l}\text { Adrienne Ettinger } \\
\text { USA }\end{array}$ & $\begin{array}{l}\text { Audra Gollenberg } \\
\text { USA }\end{array}$ & $\begin{array}{l}\text { Maggie Hendry } \\
\text { UK }\end{array}$ \\
\hline $\begin{array}{l}\text { Euzebus Ezugwu } \\
\text { Nigeria }\end{array}$ & $\begin{array}{l}\text { Brett Gordon } \\
\text { Australia }\end{array}$ & $\begin{array}{l}\text { Bernardo Hernandez } \\
\text { USA }\end{array}$ \\
\hline $\begin{array}{l}\text { Alexandre Faisal-Cury } \\
\text { Brazil }\end{array}$ & $\begin{array}{l}\text { Shelley Gorman } \\
\text { Australia }\end{array}$ & $\begin{array}{l}\text { Wendy Heywood } \\
\text { Australia }\end{array}$ \\
\hline $\begin{array}{l}\text { Jamie Feldman } \\
\text { USA }\end{array}$ & $\begin{array}{l}\text { Rebecca Grainger } \\
\text { New Zealand }\end{array}$ & $\begin{array}{l}\text { Andrew Hill } \\
\text { New Zealand }\end{array}$ \\
\hline $\begin{array}{l}\text { Deborah Fenlon } \\
\text { UK }\end{array}$ & $\begin{array}{l}\text { Catherine Granger } \\
\text { Australia }\end{array}$ & $\begin{array}{l}\text { Grace Hillyer } \\
\text { USA }\end{array}$ \\
\hline $\begin{array}{l}\text { Bradford Fenton } \\
\text { USA }\end{array}$ & $\begin{array}{l}\text { Violanda Grigorescu } \\
\text { USA }\end{array}$ & $\begin{array}{l}\text { Janet Hirst } \\
\text { UK }\end{array}$ \\
\hline $\begin{array}{l}\text { Herve Fernandez } \\
\text { France }\end{array}$ & $\begin{array}{l}\text { Marika Guggisberg } \\
\text { Australia }\end{array}$ & $\begin{array}{l}\text { Kelsey Holt } \\
\text { USA }\end{array}$ \\
\hline $\begin{array}{l}\text { Tesfaye Regassa Feyissa } \\
\text { Ethiopia }\end{array}$ & $\begin{array}{l}\text { Solomon K Gumanga } \\
\text { Ghana }\end{array}$ & $\begin{array}{l}\text { Matthew Hopkins } \\
\text { USA }\end{array}$ \\
\hline
\end{tabular}


Reihaneh Hosseini

Iran

Louise Michele Howard

UK

Ming-I Hsu

Taiwan

Vivian Huang

Canada

Megan Huchko

USA

Myra Hunter

UK

Thelma Hurd

USA

Sanusi Ibrahim

Nigeria

Nneka Iloanusi

Nigeria

Ugo Indraccolo

Italy

Mohamad Irani

USA

Mercy Isichei

Nigeria

Rafiqul Islam

Bangladesh

Lisa Iversen

UK

Nadia Jahangiri

Iran

D. Elizabeth Jesse

USA

Nattinee Jitnarin

USA

Caroline Jones

UK

Heidi Jones

USA

Esmè Jordaan

South Africa

Deborah Karasek

USA

Rajendra Karkee

Nepal
Teketo Kassaw

Ethiopia

Cihan Kaya

Turkey

Nkomba Kayeyi

Norway

Sarah Keadle

USA

Deanna Kepka

USA

Zeinab Khadr

Egypt

Anne Khasakhala

Kenya

Celso Khosa

Mozambique

Fanny Kilpi

Finland

Min Jung Kim

South Korea

Evan Kleiman

USA

M. Tish Knobf

USA

Yi-Li Ko

Taiwan

Nikolaos Kofotolis

Greece

Jung-Nien Lai

China

Rebecca Landy

UK

Katrin Lang

Estonia

Gerri Lasiuk

Canada

Pallavi Latthe

UK

Justin Lavner

USA

Angela Lawson

USA

Huynh-Nhu Le

USA
Deborah Legorreta

Mexico

Jason Leider

USA

Charlotte Lenselink

Netherlands

Iñaki Lete

Spain

Lucy Lewis

Australia

Sonia Lewycka

New Zealand

Els Leye

Belgium

Chung-Yi Li

Taiwan

Michal Liebergall

Israel

Xiong Likuan

China

Jinn Lin

Taiwan

Annika Lindskog

Sweden

Robert Liston

Canada

Farah Lone

Poland

Virginia Lope

Spain

Artur Ludwin

Poland

Kazuyo Machiyama

UK

Margaret Madeleine USA

Stéphanie Madill

Canada

Brianna Magnusson USA

Michael Johnson Mahande

Tanzania

Pranitha Maharaj

South Africa 
Mats Malqvist

Sweden

Suzanne Maman

USA

Michele Manahan

USA

Vinoj Manning

India

Roberto Marci

Italy

Desalegn Markos

Ethiopia

Morgan Marks

USA

Victoria Martinez Moron

Spain

Koji Matsuo

USA

Maria Matteo

Italy

Dimitrios Mavrelos

UK

Michael Mbizvo

Switzerland

Janet Mccabe

Canada

Angele Mcgrady

USA

Ian Mclennan

New Zealand

Bindu Mehta-Chimote

India

Andrea Mejia Rios

Colombia

Nicolas Mendoza

Spain

Melissa A. Merritt

UK

Katrina Milaney

Canada

Andrea Milbourne

USA

Karen Miller

USA
Silvia Minozzi

Italy

Suneeta Mittal

India

Bente Moen

Norway

Mitike Molla

Ethiopia

Patricia Moorman

USA

Kathryn Moracco

USA

Jane Morrell

UK

Morten Moshagen

Germany

Zulf Mughal

UK

Tamire Mulugeta

Ethiopia

Matthew Mulvey

USA

Namuunda Mutombo

Kenya

Ludovico Muzii

Italy

Shizar Nahidi

Australia

Aziza Nassar

USA

Joseph Nassif

Lebanon

Binod Neupane

Canada

Louise Newman

Australia

José Luis Neyro

Spain

Ernest Ng

Hong Kong

Enock Ngome

Botswana

Tram Nguyen

Australia
Hanh Nguyen

Viet Nam

Virginie Nicaise

France

Edgar Nieto

Venezuela

Maryam Niknejadi

Iran

Zeljka Nikolic

Serbia

Abigail Norris Turner

USA

Krzysztof Nowosielski

Poland

Theophilus Nwankwo

Nigeria

Theo Nwankwo

Nigeria

Samuel Obi

Nigeria

Shawn O'Connor

Canada

Björn Oddens

Netherlands

Kirsten Oinonen

Canada

Chinelo Okigbo

Nigeria

Ellinor Olander

UK

Margaret Olsen

USA

Suzanne O'Neill

USA

Maricianah Atieno Onono

Kenya

Livia Elisa Ortensi

Italy

Emre Pabuccu

Turkey

Manoranjan Pal

India

Santiago Palacios

Spain 


\begin{tabular}{|c|c|c|}
\hline $\begin{array}{l}\text { Parisa Parsa } \\
\text { Iran }\end{array}$ & $\begin{array}{l}\text { Grzegorz Raba } \\
\text { Poland }\end{array}$ & $\begin{array}{l}\text { Ariel Sanchez } \\
\text { Argentina }\end{array}$ \\
\hline $\begin{array}{l}\text { Harnish Patel } \\
\text { UK }\end{array}$ & $\begin{array}{l}\text { Maya Ragavan } \\
\text { USA }\end{array}$ & $\begin{array}{l}\text { Maureen Sanderson } \\
\text { USA }\end{array}$ \\
\hline $\begin{array}{l}\text { Freda Patterson } \\
\text { USA }\end{array}$ & $\begin{array}{l}\text { Lauren Ralph } \\
\text { USA }\end{array}$ & $\begin{array}{l}\text { Pavlos Sarafis } \\
\text { Greece }\end{array}$ \\
\hline $\begin{array}{l}\text { Mandira Paul } \\
\text { Sweden }\end{array}$ & $\begin{array}{l}\text { Nancy Reame } \\
\text { USA }\end{array}$ & $\begin{array}{l}\text { Ertan Saridogan } \\
\text { UK }\end{array}$ \\
\hline $\begin{array}{l}\text { Patricia Pautier } \\
\text { France }\end{array}$ & $\begin{array}{l}\text { David Reardon } \\
\text { USA }\end{array}$ & $\begin{array}{l}\text { David Sarwer } \\
\text { USA }\end{array}$ \\
\hline $\begin{array}{l}\text { Tao Peng } \\
\text { China }\end{array}$ & $\begin{array}{l}\text { Ronald Regal } \\
\text { USA }\end{array}$ & $\begin{array}{l}\text { Donald Saucier } \\
\text { USA }\end{array}$ \\
\hline $\begin{array}{l}\text { Carolyn Pepper } \\
\text { USA }\end{array}$ & $\begin{array}{l}\text { Jennifer Reingle } \\
\text { USA }\end{array}$ & $\begin{array}{l}\text { Takudzwa Sayi } \\
\text { USA }\end{array}$ \\
\hline $\begin{array}{l}\text { Robert L. Peralta } \\
\text { USA }\end{array}$ & $\begin{array}{l}\text { Philip Robinson } \\
\text { Australia }\end{array}$ & $\begin{array}{l}\text { Karen Scanlon } \\
\text { UK }\end{array}$ \\
\hline $\begin{array}{l}\text { Kath Peters } \\
\text { Australia }\end{array}$ & $\begin{array}{l}\text { Marta Rondon } \\
\text { Peru }\end{array}$ & $\begin{array}{l}\text { Channa Schmeink } \\
\text { Netherlands }\end{array}$ \\
\hline $\begin{array}{l}\text { Patrick Petignat } \\
\text { Switzerland }\end{array}$ & $\begin{array}{l}\text { Dana Roque } \\
\text { USA }\end{array}$ & $\begin{array}{l}\text { Peter Schwartz } \\
\text { USA }\end{array}$ \\
\hline $\begin{array}{l}\text { Stamatios Petousis } \\
\text { Greece }\end{array}$ & $\begin{array}{l}\text { Guri Rortveit } \\
\text { Norway }\end{array}$ & $\begin{array}{l}\text { Rebecca Sear } \\
\text { UK }\end{array}$ \\
\hline $\begin{array}{l}\text { Karen Phillips } \\
\text { Canada }\end{array}$ & $\begin{array}{l}\text { Sue Ross } \\
\text { Canada }\end{array}$ & $\begin{array}{l}\text { Katarina Sedlecky } \\
\text { Serbia }\end{array}$ \\
\hline $\begin{array}{l}\text { Jashvant Poeran } \\
\text { USA }\end{array}$ & $\begin{array}{l}\text { Alberto Rossi } \\
\text { Italy }\end{array}$ & $\begin{array}{l}\text { Laura Serrant } \\
\text { UK }\end{array}$ \\
\hline $\begin{array}{l}\text { Omero Poli-Neto } \\
\text { Brazil }\end{array}$ & $\begin{array}{l}\text { Ingrid Rowlands } \\
\text { Australia }\end{array}$ & $\begin{array}{l}\text { Omar Shaaban } \\
\text { Egypt }\end{array}$ \\
\hline $\begin{array}{l}\text { Ricki Pollycove } \\
\text { USA }\end{array}$ & $\begin{array}{l}\text { Chiara Ruini } \\
\text { Italy }\end{array}$ & $\begin{array}{l}\text { Donat Shamba } \\
\text { Tanzania }\end{array}$ \\
\hline $\begin{array}{l}\text { Nancy Poole } \\
\text { Canada }\end{array}$ & $\begin{array}{l}\text { Joseph Ruminjo } \\
\text { USA }\end{array}$ & $\begin{array}{l}\text { Larissa Shamseer } \\
\text { Canada }\end{array}$ \\
\hline $\begin{array}{l}\text { Maria Grazia Porpora } \\
\text { Italy }\end{array}$ & $\begin{array}{l}\text { Nancy Russo } \\
\text { USA }\end{array}$ & $\begin{array}{l}\text { Mridula Shankar } \\
\text { Australia }\end{array}$ \\
\hline $\begin{array}{l}\text { Oriol Porta } \\
\text { Spain }\end{array}$ & $\begin{array}{l}\text { Gideon Rutaremwa } \\
\text { Ethiopia }\end{array}$ & $\begin{array}{l}\text { Janet Shaw } \\
\text { USA }\end{array}$ \\
\hline $\begin{array}{l}\text { Jerilynn Prior } \\
\text { Canada }\end{array}$ & $\begin{array}{l}\text { Riadh Sadik } \\
\text { Sweden }\end{array}$ & $\begin{array}{l}\text { Jill Shawe } \\
\text { UK }\end{array}$ \\
\hline $\begin{array}{l}\text { Erica Prussing } \\
\text { USA }\end{array}$ & $\begin{array}{l}\text { Gianni Saguatti } \\
\text { Italy }\end{array}$ & $\begin{array}{l}\text { Estelle Sidze } \\
\text { Kenya }\end{array}$ \\
\hline $\begin{array}{l}\text { Carol Pullen } \\
\text { USA }\end{array}$ & $\begin{array}{l}\text { Daniel Salica } \\
\text { Spain }\end{array}$ & $\begin{array}{l}\text { Marushka L Silveira } \\
\text { USA }\end{array}$ \\
\hline $\begin{array}{l}\text { Alexandra Purdue-Smithe } \\
\text { USA }\end{array}$ & $\begin{array}{l}\text { Tarik Sammour } \\
\text { Australia }\end{array}$ & $\begin{array}{l}\text { Makeda Sinaga } \\
\text { Ethiopia }\end{array}$ \\
\hline
\end{tabular}


Selina Smith

USA

Mickey Sperlich

USA

Juliane Spiegler

Germany

Sheila Sprague

Canada

Flaminio Squazzoni

Israel

Lesley Stafford

Australia

Alana Steffen

USA

Elizabeth Stier

USA

Jamila Stockman

USA

Beth Sundstrom

USA

Vanphanom Sychareun

Laos

Jennifer Tabler

USA

Hiroshi Tamura

Japan

Bee Kang Tan

UK

Cara Tannenbaum

Canada

Meredith Tavener

Australia

Diana Taylor

USA

Parisa Tehranifar

USA

Louise Terry

UK

Doreth Teunissen

Netherlands

Harshad Thakur

India

Ravi Thiara

UK
David Thomas

USA

Lisa Thompson

USA

Gill Thomson

UK

Tanya Timeva

Bulgaria

Angela Todd

Australia

Elena Toffol

Finland

Giovanni A Tommaselli

Italy

Adetunji Toriola

USA

Ha Trinh

USA

Karen Troy

USA

Lisa Troy

USA

Jennifer Tsai

USA

Sibil Tschudin

Switzerland

Katherine Tumlinson

USA

Uche Umeh

Nigeria

M. Renee Umstattd Meyer USA

Ushma Upadhyay

USA

Lianne Urada

USA

Sameer Valsangkar

India

Sabine Van Den Akker

Netherlands

Olga Van Den Akker

UK

Emer Van Ryswyk

Australia
Douwe Verkuyl

Netherlands

Giovanni Veronesi

Italy

Lakshmi Vijayakumar

India

Amanda Vincent

Australia

Lorraine Walker

USA

Lewis Wall

USA

Sophie Warembourg

France

Charlotte Warren

Kenya

Melissa Watt

USA

Lori Weeks

Canada

Ram Weiss

Israel

Eliud Wekesa

Kenya

Benedict Weobong

Ghana

Sandra West

Australia

Nicole Westmarland

UK

Britta Wigginton

Australia

Mandika Wijeyaratne

Sri Lanka

Lesley Wilkes

Australia

Mark Willems

UK

Emma Williams

USA

Mellissa Withers

USA

Penny Wright

UK 
Chi-Jung Wu

Taiwan

Hongwei Xu

USA

Kapil Yadav

India

Teymoor Yary

Iran
Paul Yong

Canada

Nese Yuksel

Canada

Sofija Zagarins

USA

Deirdre Zander-Fox

Australia

\section{Chengquan Zhao}

USA

Guangyu Zhou

Germany 